\title{
Serovariedades y patrones de susceptibilidad a los antimicrobianos de cepas de Salmonella aisladas de alimentos en Cuba
}

\author{
Yamila Puig Peña, ${ }^{1}$ María Espino Hernández, ${ }^{2}$ Virginia Leyva Castillo, ${ }^{1}$ \\ Neibys Aportela López, ${ }^{1}$ Mayrin Machín Díaz ${ }^{1}$ y Perla Soto Rodríguez ${ }^{1}$
}

Forma de citar Puig Peña Y, Espino Hernández M, Leyva Castillo V, Aportela López N, Machín Díaz M, Soto Rodríguez P. Serovariedades y patrones de susceptibilidad a los antimicrobianos de cepas de Salmonella aisladas de alimentos en Cuba. Rev Panam Salud Publica. 2011;30(6):561-5.

RESUMEN Se describen los serotipos de 178 cepas de Salmonella enterica aisladas de alimentos en diferentes regiones de Cuba entre enero de 2008 y diciembre de 2009, y el patrón de susceptibilidad a los antimicrobianos de 100 aislados seleccionados mediante muestreo por estratos. Se identificaron 20 serovariedades de Salmonella entre las que predominaron S. Enteritidis (23\%); S. Agona (13,5\%) y S. London (11,2\%). Del total, $75 \%$ de las cepas fueron resistentes o presentaron resistencia intermedia a al menos uno de los fármacos probados, en el siguiente orden, según su frecuencia: tetraciclina (70,7\%); ampicilina (22,7\%) y ácido nalidixico (14,7\%). Se identificaron 10 patrones de resistencia diferentes y predominaron las cepas resistentes o con resistencia intermedia a un fármaco (89,3\%). Tres cepas (S. Infantis, S. Derby y S. Enteritidis) fueron multirresistentes y una, de S. Enteritidis, dio un resultado no sensible al ácido nalidíxico y la ciprofloxacina. Se destaca la necesidad de extremar la vigilancia sanitaria integrada en el país para el control de la salmonelosis.

Palabras clave Farmacorresistencia bacteriana; Salmonella enterica; farmacorresistencia bacteriana múltiple; Cuba.

La bacteria aislada con mayor frecuencia en casos de enfermedad de transmisión alimentaria (ETA) en el mundo corresponde al género Salmonella, que causa casi la mitad de los casos que se diagnostican. Un informe de la Autoridad Europea de Seguridad Alimentaria (EFSA) y del Centro Europeo de Prevención y Control de Enfermedades (ECDC) señala que esa bacteria es la causa primaria de ETA

\footnotetext{
1 Instituto de Nutrición e Higiene de los Alimentos (INHA), Departamento de Microbiología, La Habana, Cuba. Dirigir la correspondencia a: yamila@sinha.sld.cu

2 Escuela Latinoamericana de Medicina, Departamento de Microbiología y Parasitología Médica, La Habana, Cuba.
}

en dicha región (1). En los Estados Unidos de América, un informe reciente (2) indica que las cepas de Salmonella no tifoídica fueron el segundo agente patógeno más frecuente (después del norovirus) aislado del total de 9,4 millones de episodios de ETA diagnosticados en el período 2000-2008 en ese país, y que este agente patógeno es la causa principal de las hospitalizaciones por ETA. En Cuba, en el período de 2004 a 2006, se notificaron 472 brotes de salmonelosis que afectaron a 14430 personas. Los alimentos involucrados con mayor frecuencia fueron las carnes y sus derivados, en particular las de ave, los productos elaborados con carne deshuesada mecánicamente y las ensaladas frías con mayonesa elaborada con huevos crudos (3).

La ecología compleja de las cepas de Salmonella, la ubicuidad del microorganismo y la falta de signos externos que indiquen su presencia en los alimentos son los principales obstáculos para el control de la enfermedad. Por ello se hace necesario mejorar la capacidad y competencia para detectar y caracterizar los brotes mediante la identificación, tipificación sistemática y determinación de los perfiles de susceptibilidad a los antimicrobianos del microorganismo, con el fin de tomar medidas de control apropiadas. En el Instituto de Nutrición e Higiene de los Alimentos (INHA) de 
Cuba, desde 2004 se realiza un seguimiento estrecho de la susceptibilidad a los antimicrobianos de los aislamientos de Salmonella y otros agentes patógenos causantes de ETA.

El presente trabajo tuvo como objetivo determinar los serotipos y el patrón de susceptibilidad a los antimicrobianos de un grupo de aislamientos de Salmonella procedentes de una variedad de alimentos estudiados como causa de brotes por la vigilancia epidemiológica y el registro sanitario de alimentos.

La investigación abarcó el período desde enero de 2008 a diciembre de 2009. Se analizaron 178 cepas de Salmonella procedentes de diferentes regiones de Cuba, que fueron nuevamente identificadas y serotipificadas en el Laboratorio de Microbiología del INHA, según la norma cubana NC-ISO 6579: 2008 (4). Para su traslado al INHA, los aislados se conservaron en medio de agar triptona semisólido y luego se transfirieron a medio de enriquecimiento de caldo peptona de soja y agar MacConkey (BioCen, Cuba). La caracterización bioquímica se realizó según el esquema de identificación establecido por métodos de cultivo convencional. El estudio serológico se efectuó por pruebas de aglutinación en lámina con antisueros somáticos y fla- gelares de Salmonella (PRO-LAB, UK). Mediante un muestreo por estratos, se seleccionó un total de 100 cepas para realizar las pruebas de sensibilidad a los antimicrobianos; para ello se tuvo en cuenta su procedencia según el motivo del ensayo (brote de ETA, vigilancia epidemiológica y registro sanitario de alimentos) y el tipo de alimento. El método empleado fue el de difusión con discos (Bauer-Kirby) según las normas del Instituto de Estándares Clínicos y de Laboratorio (CLSI, por su sigla en inglés) (5). Los discos de antimicrobianos procedían de la casa comercial CPM-SCIENTIFICA (Roma, Italia) y fueron: ácido nalidíxico (NAL), ampicilina (AMP), ceftriaxona (CRO), ciprofloxacina (CIP), cloranfenicol (CHL), gentamicina (GEN), kanamicina (KAN), sulfametoxazol/trimetoprima (SXT) y tetraciclina (TCY). Para el control de la calidad se utilizaron las cepas: Staphylococcus aureus ATCC 25923 y Escherichia coli ATCC 25922. Se clasificaron como multirresistentes los microorganismos resistentes o con resistencia intermedia a tres o más antimicrobianos. La base de datos se conformó en el programa WHONET 5.4.

Del total de 178 aislamientos, 123 $(69,1 \%)$ se obtuvieron por la vigilancia epidemiológica: $114(92,7 \%)$ de carnes crudas, elaboradas y derivados cárnicos y $9(7,3 \%)$ de otras fuentes. Por el registro sanitario de alimentos se aislaron 16 muestras (9\%): $15(93,7 \%)$ de alimentos cárnicos y una $(6,3 \%)$ de un producto vegetal (especia). Como causa de brote se identificaron $39(21,9 \%)$ cepas aisladas principalmente de alimentos elaborados con huevo (30 aislados o 76,9\%), platos elaborados (5 aislados o 12,8\%) y carnes procesadas y derivados (cuatro aislados o 10,3\%). Se identificaron 20 serovariedades diferentes de Salmonella enterica, de las cuales las más frecuentes fueron S. Enteritidis (23\%), S. Agona $(13,5 \%)$ y $S$. London (11,8\%) (cuadro 1).

Estos resultados coinciden con trabajos consultados (6-9) que muestran que los aislamientos de $S$. Enteritidis constituyen la serovariedad más frecuente. Por otra parte, en Cuba se han notificado casos de salmonelosis por S. Agona (10). De modo similar a lo informado por UNICEF/Cuba en el período 2004-2006, (3) los aislamientos de Salmonella se obtuvieron principalmente de carnes crudas o procesadas y de alimentos elaborados con huevo. Resultó llamativo el hallazgo de una cepa de $S$. Typhimurium en una especia, lo que es indicativo de la diversidad de alimentos en los que es posible encontrar la bacteria y la importancia

CUADRO 1. Serovariedades de cepas de Salmonella enterica identificadas y alimento de procedencia, Instituto Nacional de Higiene de los Alimentos, La Habana, Cuba, 2008 y 2009

\begin{tabular}{|c|c|c|c|c|c|c|c|c|c|}
\hline Serotipos de Salmonella & $\begin{array}{l}\text { Carne } \\
\text { fresca }\end{array}$ & $\begin{array}{c}\text { Carne } \\
\text { elaborada }\end{array}$ & $\begin{array}{l}\text { Productos } \\
\text { elaborados } \\
\text { con carne }\end{array}$ & $\begin{array}{l}\text { Postres y otros } \\
\text { alimentos a base } \\
\text { de huevos }\end{array}$ & $\begin{array}{c}\text { Platos } \\
\text { elaborados }\end{array}$ & Lácteos & Especias & Total & $\%$ \\
\hline S. Agona & 16 & 4 & 3 & 1 & 0 & 0 & 0 & 24 & 13,5 \\
\hline S. Amsterdan & 1 & 2 & 2 & 1 & 0 & 0 & 0 & 6 & 3,4 \\
\hline S. Anatum & 4 & 1 & 6 & 0 & 0 & 0 & 0 & 11 & 6,2 \\
\hline S. Asylanta & 0 & 1 & 1 & 0 & 0 & 0 & 0 & 2 & 1,1 \\
\hline S. Bissau & 9 & 1 & 1 & 0 & 0 & 0 & 0 & 11 & 6,2 \\
\hline S. Derby & 7 & 1 & 6 & 1 & 0 & 0 & 0 & 15 & 8,4 \\
\hline S. Enteritidis & 6 & 0 & 10 & 23 & 2 & 0 & 0 & 41 & 23,0 \\
\hline S. Hato & 2 & 0 & 0 & 0 & 0 & 0 & 0 & 2 & 1,1 \\
\hline S. Infantis & 5 & 2 & 1 & 1 & 0 & 2 & 0 & 11 & 6,2 \\
\hline S. Irumu & 0 & 0 & 0 & 1 & 0 & 0 & 0 & 1 & 0,6 \\
\hline S. Kande & 0 & 1 & 0 & 0 & 0 & 0 & 0 & 1 & 0,6 \\
\hline S. Kisii & 1 & 0 & 0 & 1 & 0 & 0 & 0 & 2 & 1,1 \\
\hline S. London & 10 & 1 & 6 & 2 & 2 & 0 & 0 & 21 & 11,8 \\
\hline S. Montevideo & 0 & 1 & 1 & 1 & 0 & 0 & 0 & 3 & 1,7 \\
\hline$S$. Muenchen & 5 & 0 & 0 & 2 & 0 & 0 & 0 & 7 & 3,9 \\
\hline S. Muenster & 1 & 1 & 0 & 0 & 0 & 0 & 0 & 2 & 1,1 \\
\hline S. Nchanga & 2 & 0 & 0 & 0 & 0 & 0 & 0 & 2 & 1,1 \\
\hline S. Shangani & 2 & 0 & 0 & 0 & 0 & 0 & 0 & 2 & 1,1 \\
\hline S. Sinstorf & 1 & 0 & 3 & 1 & 0 & 0 & 0 & 5 & 2,8 \\
\hline S. Typhimurium & 4 & 0 & 1 & 2 & 1 & 0 & 1 & 9 & 5,1 \\
\hline Total (\%) & $76(42,7)$ & $16(9,0)$ & $41(23,0)$ & $37(20,8)$ & $5(2,8)$ & $2(1,1)$ & $1(0,6)$ & 178 & 100,0 \\
\hline
\end{tabular}


de realizar la vigilancia epidemiológica de otros alimentos, como vegetales y frutas. Informes recientes de los Centros para el Control y Prevención de las Enfermedades de los Estados Unidos (11, 12) destacan la ocurrencia de brotes de salmonelosis asociados al consumo de frutas frescas.

Las pruebas de sensibilidad a los antimicrobianos indicaron que del total de 100 cepas estudiadas, $75(75 \%)$ fueron resistentes o presentaron resistencia intermedia a al menos uno de los nueve fármacos probados. De esas 75, el fármaco al cual hubo mayor resistencia fue tetraciclina (53 cepas o 70,7\%), seguido de ampicilina (17 cepas o $22,7 \%$ ), ácido nalidíxico (11 cepas o 14,7\%); ceftriaxona (4 ó 5,3\%) y ciprofloxacina (1 ó 1,3\%). Se identificaron 10 patrones de resistencia diferentes. Así, con mayor frecuencia se detectó resistencia o resistencia intermedia a un único fármaco (67 aislamientos, $89,3 \%)$. De estas 67 muestras, $47(62,7 \%)$ fueron resistentes a TCY; $12(16 \%)$ a AMP y $6(8 \%)$ a NAL. Se destaca el hallazgo de tres aislamientos multirresistentes con patrones AMP-TCY-CRO (S. Infantis aislada de carne fresca) y AMP-TCY-NAL ( $S$. Derby y $S$. Enteritidis aisladas de productos elaborados con carne y huevo, respectivamente). También se detectaron cuatro aislados con susceptibilidad disminuida a ceftriaxona y una cepa de $S$. Enteritidis no sensible a quinolonas (patrón NAL-CIP) (cuadro 2).

Lo encontrado en este estudio coincide con lo informado en otros trabajos realizados en el país y otras regiones (8-10), acerca de la resistencia elevada a tetraciclina y ampicilina, que generalmente se atribuye al amplio uso que durante décadas han tenido esos medicamentos, tanto en medicina humana como veterinaria. La resistencia identificada en este trabajo podría estar relacionada con las prácticas utilizadas en las explotaciones pecuarias en el país, si se tiene en cuenta que las principales fuentes de alimentos involucradas son de origen animal. El uso de antibióticos para promover el crecimiento de los animales de abasto y en algunos cultivos son prácticas muy antiguas utilizadas en todo el mundo y se han relacionado con el desarrollo acelerado de la resistencia a los antimicrobianos. En Cuba, las autoridades veterinarias indican que no se debe emplear antibióticos en el engorde de los anima-

CUADRO 2. Patrones de resistencia de las cepas de Salmonella no sensibles, según serotipo y alimento de origen, Instituto Nacional de Higiene y Alimentos, La Habana, Cuba, 2008-2009

\begin{tabular}{|c|c|c|c|}
\hline Serotipo & No. de cepas & Patrón & Fuente de aislamiento \\
\hline S. Enteritidis & 18 & TCY & $\begin{array}{l}\text { Carne fresca } \\
\text { Productos a base de huevo } \\
\text { Productos elaborados con carne }\end{array}$ \\
\hline S. Typhimurium & 4 & TCY & $\begin{array}{l}\text { Carne fresca } \\
\text { Especia }\end{array}$ \\
\hline S. Agona & 3 & TCY & $\begin{array}{l}\text { Carne fresca } \\
\text { Carne elaborada }\end{array}$ \\
\hline S. Anatum & 3 & TCY & $\begin{array}{l}\text { Carne fresca } \\
\text { Productos elaborados con carne }\end{array}$ \\
\hline S. Infantis & 3 & TCY & Carne fresca \\
\hline S. Asylanta & 2 & $\mathrm{TCY}$ & Productos elaborados con carne \\
\hline S. Derby & 2 & TCY & Carne fresca \\
\hline S. Kisii & 1 & TCY & Productos a base de huevo \\
\hline S. Muenchen & 2 & TCY & Carne fresca \\
\hline S. Nchanga & 2 & $\mathrm{TCY}$ & Carne fresca \\
\hline S. Sinstorf & 2 & TCY & Productos elaborados con carne \\
\hline S. Hato & 1 & TCY & Carne fresca \\
\hline S. Irumu & 1 & TCY & Productos a base de huevo \\
\hline S. Kande & 1 & TCY & Carne elaborada \\
\hline S. Montevideo & 1 & TCY & Carne elaborada \\
\hline S. Shangani & 1 & TCY & Carne fresca \\
\hline S. Derby & 1 & AMP & Carne fresca \\
\hline S. London & 2 & AMP & Carne fresca \\
\hline S. Typhimurium & 2 & AMP & Productos a base de huevo \\
\hline S. Infantis & 3 & AMP & Productos lácteos \\
\hline S. Enteritidis & 4 & AMP & Productos a base de huevo \\
\hline S. Bissau & 1 & NAL & Carne fresca \\
\hline S. London & 1 & NAL & Carne fresca \\
\hline S. Agona & 2 & NAL & Carne fresca \\
\hline S. Enteritidis & 2 & NAL & Carne elaborada \\
\hline S. Typhimurium & 1 & $\mathrm{CRO}$ & Carne fresca \\
\hline S. Agona & 1 & CRO & Carne fresca \\
\hline S. Typhimurium & 1 & AMP-TCY & Carne fresca \\
\hline S. London & 1 & AMP-TCY & Carne fresca \\
\hline S. Kisii & 1 & NAL-TCY & Carne fresca \\
\hline S. London & 1 & NAL-CRO & Carne fresca \\
\hline S. Enteritidis & 1 & NAL-CIP & Carne fresca \\
\hline S. Derby & 1 & AMP-TCY-NAL & Productos elaborados con carne \\
\hline S. Enteritidis & 1 & AMP-TCY-NAL & Productos a base de huevo \\
\hline S. Infantis & 1 & AMP-TCY-CRO & Carne fresca \\
\hline Total & 75 & & \\
\hline
\end{tabular}

TCY: Tetraciclina, AMP: ampicilina, NAL: ácido nalidíxico, CRO: ceftriaxona, CIP: ciprofloxacina.

les, aunque, no contamos con informes relacionados con estas recomendaciones.

El hallazgo de cuatro cepas con susceptibilidad disminuida a ceftriaxona es una advertencia acerca de la posible presencia en nuestro medio de bacterias del género Salmonella productoras de enzimas BLEE $u$ otras $\beta$-lactamasas de amplio espectro ya notificadas por otros autores (13). También despierta interés la identificación de la cepa $S$. Enteritidis no sensible a quinolonas, pues ello podría significar la extensión en nuestro medio de un clon resistente a estos fármacos, ya que $14,7 \%$ de las cepas estudiadas dieron resultado no sensible al ácido nalidíxico. Esta proporción se considera elevada ante la amplia utilidad terapéutica de las quinolonas para el tratamiento de las salmonelosis y otras infecciones de origen bacteriano. La resistencia de las cepas de Salmonella al ácido nalidíxico y su susceptibilidad reducida a las fluroquinolonas se han notificado en otros estudios (14).

Desde hace más de 50 años, se trabaja en Cuba en el establecimiento de una estrategia nacional para el control de las enfermedades infecciosas que pueden afectar al ser humano y otros animales. 
En el campo de la higiene y la epidemiología y la medicina veterinaria, se han tomado medidas preventivas para garantizar la calidad de los productos de origen animal, con el objetivo de lograr la inocuidad de estos alimentos (15). Los diversos servicios poseen una red diagnóstica en distintos ámbitos geográficos (municipal, provincial y nacional) que cuentan con equipos de inspectores encargados de controlar el estado higiénico y sanitario de los alimentos. No obstante, se considera necesario promover una vigilancia integrada. Para el control de la salmonelosis, en particular, se hace necesario desarrollar una vigilancia de la cadena alimentaria que tenga como punto de partida la identificación de todas las posibles vías por las cuales las cepas de Salmonella puedan llegar a los alimentos. Asimismo, es necesario investigar las fases de producción de los alimentos, fundamentalmente los cárnicos, y tener en cuenta todas las etapas del procesa- miento, desde la materia prima hasta el producto final elaborado. También habrá que lograr la determinación etiológica específica que incluya los serotipos y antibiotipos, conjuntamente con la identificación de los posibles reservorios en la población.

La falta de recursos de diagnóstico para determinar los patrones de susceptibilidad de todas las cepas involucradas fue la limitante principal de esta investigación. Se prevé resolver esta situación en trabajos futuros con una planificación que permita aumentar el número de cepas que se estudien y la inclusión de procedimientos específicos para corroborar, entre otros, la presencia de enzimas BLEE.

Como conclusión, se determinó que en los alimentos analizados predominó la presencia de los serotipos $S$. Enteritidis, $S$. Agona y $S$. London. Las carnes y los productos elaborados con carne analizados, principalmente como parte de la vigilancia epidemiológica, y los productos elaborados con huevos, en su mayoría vinculados a brotes, fueron las principales fuentes de los aislamientos. La tetraciclina, la ampicilina y el ácido nalidíxico constituyeron los antibióticos ante los cuales hay menos sensibilidad. Predominó el patrón de resistencia a un solo fármaco, aunque también se detectaron cepas multirresistentes.

En la producción primaria de animales de abasto, se recomienda brindar especial atención al empleo de los antibióticos, como tetraciclina, ampicilina y ácido nalidíxico, ya sea para tratar o prevenir enfermedades; trabajar en el mejoramiento de la recopilación y utilización sistemática de la información relacionada con los marcadores epidemiológicos abordados, para lograr una mejor trazabilidad de los serotipos de Salmonella circulantes y de los perfiles de susceptibilidad según fuente de aislamiento y regiones del país. Esto mejorará las medidas para el control y la prevención de la salmonelosis.

\section{REFERENCIAS}

1. Autoridad Europea de Seguridad Alimentaria (EFSA). [Internet]. Informe de la EFSA-ECDC sobre intoxicaciones alimentarias en la UE 2007. Disponible en: http:/ / www.higieneambiental.com/ higiene-alimentaria / informe-de-efsaecdc-sobre-intoxicaciones-alimentariasen-la-ue-2007 Acceso el 29 de agosto de 2011.

2. Scallan E, Hoekstra RM, Angulo FJ, Tauxe RV, Widdowson M-A, Roy SL, et al. Foodborne illness acquired in the United Statesmajor pathogens. Emerg Infect Dis [serial on the Internet]. 2011 Jan; http://dx.doi. org/10.3201/eid1701.P11101

3. UNICEF/Cuba [Internet]. Vigilancia de contaminantes biológicos (Salmonella) en alimentos específicos. Disponible en: http://www. unicef.cu / cuaternario.php?id $=04 \&$ id menu $=04 \_03 \&$ data $=04 \_03 \_08 \&$ iddata $=$ 04_03_08_01 Acceso el 29 de agosto de 2011.

4. Microbiología de alimentos de consumo humano y animal-Guía general para la detección de Salmonella. NC ISO 6579. 2008.

5. Clinical and Laboratory Standards Institute (CLSI). Performance standards for antimicrobial susceptibility testing: Sixteenth Informational Supplement. 2008;26(3). M100-S16.

6. Ran L, Wu S, Gao Y, Zhang X, Feng $Z$, Wang $Z$, et al. Laboratory-based surveillance of nontyphoidal Salmonella infections in China. Foodborne Pathog Dis. 2011;8(8):921-7. Epub 2011 Apr 14.
7. Miller AJ, Twomey DF, Davies RH, Teale CJ, Williamson SM, Reichel R, et al. Salmonella serovars and antimicrobial resistance patterns on a sample of high seroprevalence pig farms in England and Wales (20032008). Zoonoses and Public Health. doi: 10.1111/j.1863-2378.2011.01402.x.

8. Kim S. Salmonella serovars from foodborne and waterborne diseases in Korea, 1998-2007: total isolates decreasing versus rare serovars emerging. J Korean Med Sci. 2010;25(12):1693-99.

9. Public Health Agency of Canada [Internet]. National Microbiology Laboratory. 2009 Annual Summary of Laboratory Surveillance Date. Appendix 1. Serotype data reported to NESP in 2009. Disponible en: http:// www. nml-lnm.gc.ca / NESP-PNSME/ surveil lance-2009-app1-eng.html Acceso el 29 de agosto de 2011.

10. Cabrera R, Ruiz J, Ramírez M, Bravo L, Fernández A, Aladueña A, et al. Dissemination of Salmonella enterica serotype Agona and multidrug-resistant Salmonella enterica serotype Typhimurium in Cuba. An J Trop Med Hyg. 2006;74(6):1049-53.

11. Centers for Disease Control and Prevention. CDC. [Internet]. Atlanta: Investigation Announcement: Multistate outbreak of human Salmonella Agona infections linked to whole, fresh imported papayas. Disponible en: http://www.cdc.gov/salmonella / agona/ Acceso el 29 de agosto de 2011.
12. Centers for Disease Control and Prevention CDC. [Internet]. Investigation of outbreak of infections caused by Salmonella Litchfield. Disponible en: http://www.cdc.gov/ salmonella/litchfield/ Acceso el 29 de agosto de 2011.

13. Madec JY, Doublet B, Ponsin C, Cloeckaert A, Haenni M. Extended-spectrum beta-lactamase blaCTX-M-1 gene carried on an IncI1 plasmid in multidrug-resistant Salmonella enterica serovar Typhimurium DT104 in cattle in France. J Antimicrob Chemother. 2011;66(4):942-4.

14. Kim KY, Park JH, Kwak HS, Woo GJ. Characterization of the quinolone resistance mechanism in foodborne Salmonella isolates with high nalidixic acid resistance. Int J Food Microbiol. 2011;146(1):52-6.

15. Estrada CO, Castro MD, Rosales VA, González RO, Tamayo MN. Comparación de la efectividad de la norfloxacina y la oxitetraciclina en el tratamiento de la colibacilosis en aves. VET-UY [Internet] Jun 2009 Disponible en: http://www.vet-uy.com/ articulos/avicultura/100/051/avic051. htm Acceso el 29 de agosto de 2011

Manuscrito recibido el 8 de abril de 2011. Aceptado para publicación, tras revisión, el 23 de septiembre de 2011. 
ABSTRACT The serotypes of 178 isolates of Salmonella enterica taken from food in different regions of Cuba between January 2008 and December 2009 were identified, and the antimicrobial susceptibility pattern of 100 selected isolates was determined by strata

Serotypes and antimicrobial susceptibility patterns of Salmonella strains isolated from food in Cuba

Key words sampling. A total of 20 Salmonella serotypes were identified, with a predominance of S. Enteritidis (23\%), S. Agona (13.5\%), and S. London (11.2\%). Of all the strains, $75 \%$ were resistant or presented intermediate resistance to at least one of the drugs tested, in the following order: tetracycline $(70.7 \%)$, ampicillin $(22.7 \%)$, and nalidixic acid $(14.7 \%)$. Ten different resistance patterns were identified. The most frequent patterns corresponded to strains that were either drug-resistant or had intermediate resistance (89.3\%). Three strains (identified as S. Infantis, S. Derby, and S. Enteritidis) were multiresistant, and one of them, $S$. Enteritidis, was not sensitive to either nalidixic acid or ciprofloxacin. To control salmonellosis, the importance of maximizing integrated health surveillance is emphasized.

Drug resistance, bacterial; Salmonella enterica; drug resistance, multiple, bacterial; Cuba. 